\title{
Robust Algorithm for Heart Rate (HR) Detection and Heart Rate Variability (HRV) Estimation
}

\author{
Z. Piotrowski ${ }^{a}$ AND K. RÓŻANOWSKI ${ }^{b}$ \\ ${ }^{a}$ Telecommunications Institute, Military University of Technology \\ Kaliskiego 2, 00-908 Warsaw, Poland \\ ${ }^{b}$ Military Institute of Aviation Medicine, Krasińskiego 54, 01-455 Warsaw, Poland
}

\begin{abstract}
We present algorithm for Heart Rate detection based on Short-Term Autocorrelation Center Clipping method. This algorithm is dedicated for biological signal detection, electrocardiogram, in noisy environment with lot of artifacts. Using this algorithm is also possible detect the $\mathrm{R}$ pointers in the PQRST complex of the ECG signal. In this paper the new implementation of the heart rate variability estimation is also presented. HRV module is based on parametric and non-parametric methods of the power spectral density computation.
\end{abstract}

PACS numbers: 87.85.Ng, 87.19.Le, 84.37.+q, 87.57.C-

\section{Introduction}

This paper presents algorithm for Heart Rate (HR) detection used in electrocardiogram monitoring system as well as algorithm for Heart Rate Variability (HRV) estimation. During the training dedicated flights, military pilots are monitored for checking their susceptibility to stress and self-control. ECG data-logger as a personal portable device is collecting biological signal during the testing flight. Computed HR values together with Accumulated Reference Pattern PQRST complex (ARP) are estimates of pilot's health. ECG analysis is performed using Short-term Autocorrelation Center Clipping (SACC) method. SACC method dedicated for HR detection and ECG R-pointers analysis is very robust for the noisy environment. HRV parameters estimation is based on advanced spectral analysis of electrocardiogram (tachogram of $R R$ values). HRV estimation is noninvasive method of estimation the Sympathetic and Parasympathetic Nervous System influence on the heart rate.

\section{Decontraction of the heart muscule - rate detection problem}

In the case of presence both high level noise in the biological signal background and large number of artifacts it is considered very high degradation useful for analysis purposes ECG signal [1-11]. Most known algorithms used for R wave of ECG detection are not robust against ECG signal acquisition trials in the case of patient's movement, electrodes' displacement as well as during work in increased electromagnetic radiation.

These all adverse conditions, from acquisition process point of view, appears during pilot's combat aircraft flight. ECG degradation factors are: pilot's relocating in the cockpit caused by sustained gravity load and in progress operations on aircraft board, identification and active boarding radiolocation systems switched on as well as others devices generating electromagnetic field.

\subsection{Heart Rate detection algorithm}

HR detection algorithm [12] consists of two modules developed in the form of dynamic linked library (*.dll) entitled Signal Analysis Module (pol. Modut Analizy Przebiegów, MAP) accordingly: MAP-A (for R waves ECG detection considered as a set of indexes of $\mathrm{R}$ waves occurrence and $\mathrm{HR}$ computation based on indexes of $\mathrm{R}$ waves ECG), and MAP-B (Accumulated Reference Pattern PQRS complex computation).

MAP-A block consist of four modules: mapa01-mapa04 responsible for following procedures:

- mapa01 - ECG signal's acquisition (low-pass filtering, long-term trend removing, high-pass filtering, amplitude equalization and adaptive power correction),

- mapa02 - HR computation using Short-term Autocorrelation Center Clipping method (SACC),

- mapa03 - determination an R wave ECG indexes based on HR computation,

- mapa04 - HR computation based on determined an R wave ECG indexes, Power Spectral Density (PSD) estimation.

Mapa01 block consist of several modules, scheme is shown at the Fig. 1.

Mapa 01 block is devided in five processing stages (blocks: mapa01A-01E). Following stages are carried out: 
ECG signal with sampling frequency $f_{s}=250 \mathrm{~Hz}$ is filtered (low-pass filter with cut-off frequency $f_{c}=124 \mathrm{~Hz}$ ), long-term trend is removed using window with length LeFrm $=250$ samples, signal is averaging using Finite Impulse Response Averaging Filter (FIR Avg) with order $N=9$, amplitude is equalized (AmpCorr) using normalized coefficient and adaptive power correction is used (AGC).

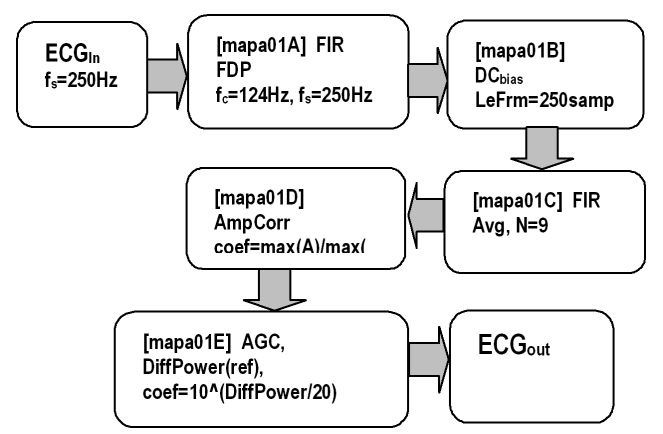

Fig. 1. Signal Processing basic scheme in mapa01 module.

Biological ECG signal is acquired with sampling frequency rate $f_{s}=250 \mathrm{~Hz}$. In the block mapa01 A ECG signal is filtering using FIR low-pass filter with cut-off frequency $f_{c}=124 \mathrm{~Hz}$ and order $N$ equals 700 . Thus maximum acquired signal's frequency for $f_{\mathrm{N}}=f_{s} / 2=125 \mathrm{~Hz}$ is $124 \mathrm{~Hz}$, with relatively low ripples in the pass-band $\left(R_{p}=0.1 \mathrm{~dB}\right)$, low transient band, high suppression in the stop-band $\left(R_{s}=80 \mathrm{~dB}\right)$ as well as linear phase in the pass-band. Designed FIR filter is always stable considering filter's impulse response.

In the mapa01B block signal is detrended (long-term trend removing). Signal's trend is a systematical error loading acquired ECG signal by increasing constant component. Trend is removed by subtracting from each signal's sample mean amplitude value of signal's window (250 samples length). In the mapa01C block signal is processing using FIR, $N=9$ order, moving average filter. Signal averaging procedure significantly decreasing signal's amplitude, it is not desired effect but at the same time higher frequency components are reduced too.

In the mapa01D block amplitude correction is carried out to reach amplitude value before MA filtering. Correction coefficient is computed using following formula (1) and correction result is shown in (2):

$$
\begin{aligned}
& \text { coef }=\frac{\max (\operatorname{FrmMA})}{\max (\operatorname{Frm} D C)}, \\
& \text { FrmMAcorr }=\text { coef } \times \text { FrmDC, }
\end{aligned}
$$

where: FrmMA - signal frame after MA correction, FrmDC - signal frame before MA correction, FrmMAcorr - signal's frame value after amplitude correction.

In the mapa01E block is used adaptive power correction procedure. Main aim of this procedure is to reduce standard deviation of the acquired ECG signal. In this way temporary disturbances (artifacts) are smoothed e.g. too big electrode's resistance, temporary electrode's displacement. The only these signal's frames are corrected which power value exceed fixed threshold limit (3). Reference frame refFrm is chosen as a computed median of a standard deviation from a set of all frames.

$$
\text { refPower }=3 \sum_{i=1}^{M} \text { refFrm }_{i, \text { median_std }}^{2},
$$

where: $i$ - sample number in reference frame, $M$ number of samples in reference frame, refFrm - frame (set of 250 samples).

Amplitude correction for frame (set of samples) is processed by adaptive frame power decreasing per $1 \mathrm{~dB}$ up to the iteration when power of corrected frame achieve the lower or equal reference value.

Main task of the mapa02 block in MAP-A module is Heart Rate computation. We use Short-term Autocorrelation Center Clipping (SACC) procedure for this purpose. Based on computed periods of $R$ wave ECG in module mapa03 $\mathrm{R}$ indexes are detected. We use classic tri-drain ECG system where following electrodes have names: ecg1, ecg2, ecg3. Algorithm's procedures take into account all configurations (electrode accessibility) e.g. accessible are following signals: ecg2 and ecg3, then algorithm computes HR for signal ecg2, taking advantage of ecg3 in the case of strong artifacts and disturbances. Another case: accessible are all ecg signals 1-3 and ecg2 signal is strongly degraded then algorithm detects this fact and takes into account only ecg1 and ecg3 signals for HR detection. Tri-stage processing means that in case when all ecg signals $1-3$ are given in input mapa02 block, then ecg1 signal will be considered as a base for analysis and in the case of strongly degraded fragments, other signals (ecg2-3) will be processed, switched in turn the SACC searching range.

Used method of $\mathrm{R}$ wave ECG periods signal searching based on short-term autocorrelation function. At Fig. 2 is shown the result of short term autocorrelation function of ECG signal in 750 samples length. Autocorrelation function is normalized and clipped using thresholds $\alpha=$ 0.45 and $\beta=-0.45$ with following formula:

$$
C\{s(n)\}\left\{\begin{array}{c}
s(n)-\alpha, s(n)>\alpha, \\
0, \beta \leq s(n) \leq \alpha, \\
s(n)-\beta, s(n)<\beta .
\end{array}\right.
$$

The result of normalization and clipping is shown at Fig. 3.

Distance between first sample and maximum sample of the first harmonic determines period of R waves ECG, thus it is possible to determine $\mathrm{HR}$ in beats per minutes (bpm) using following formula:

$$
H R_{\text {bpm }}=\frac{60 * 250}{\text { FoundIndex }},
$$

where: FoundIndex — distance in number of samples between max sample of SACC and max sample of the first harmonic.

Frame length equals 750 samples, thus each frame represents 3 seconds of signal (assumed: $f_{s}=250 \mathrm{~Hz}$ ). We 


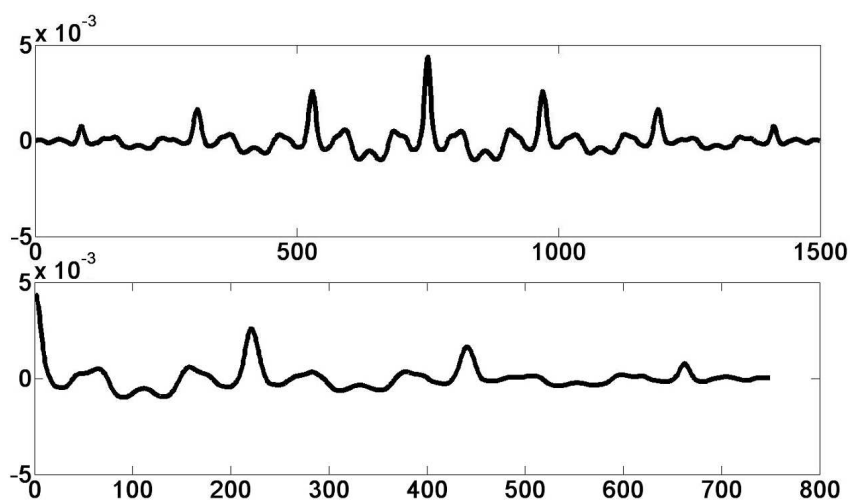

Fig. 2. Short term autocorrelation function for ecg signal (x-axis - number of samples, y-axis amplitude values).

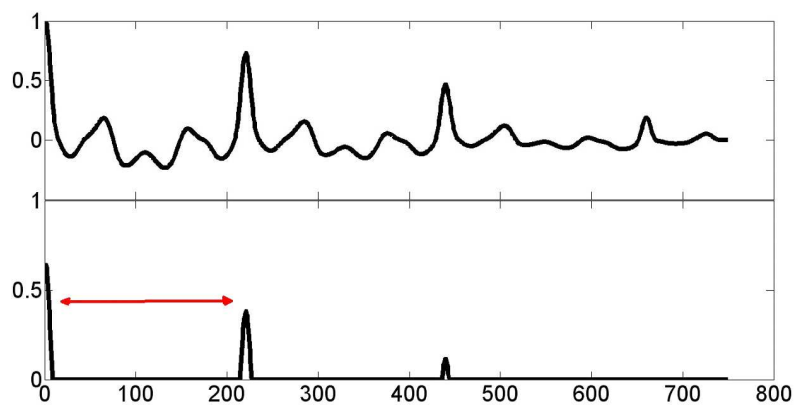

Fig. 3. Effect of autocorrelation processing after clipping and normalization ( $\mathrm{x}$-axis - number of samples, $\mathrm{y}$-axis amplitude values).

assumed that range of possible $\mathrm{RR}$ period is between values: 50 and 750 then $\mathrm{HR}$ range is form $300 \mathrm{bpm}$ to $20 \mathrm{bpm}$.

\subsection{Accumulated reference pattern (ARP) PQRST complex}

MAP-B module based on pre-processed ECG signal in the mapa01 block. PQRST complex segmentation is a first stage of the signal processing. In this processing indexes of $\mathrm{R}$ waves ECG signal are used. At Fig. 4. example of ECG route with detected $\mathrm{R}$ waves is shown.

$\mathrm{R}$ pointers are used for PQRST complexes segmentation. $\mathrm{R}$ pointers are detected in mapa03 block. Each PQRST complex is buffered and value of its length is computed. In the sorting procedure, PQRST complexes possessing the same length are grouped. For the next processing stage following PQRST complexes are chosen:

(a) the same complexes possessing the most frequently occurred length,

(b) the same complexes as a median period value from all period values.

These two groups of PQRST complexes are coherent averaged. During averaging process the Coherence Gain

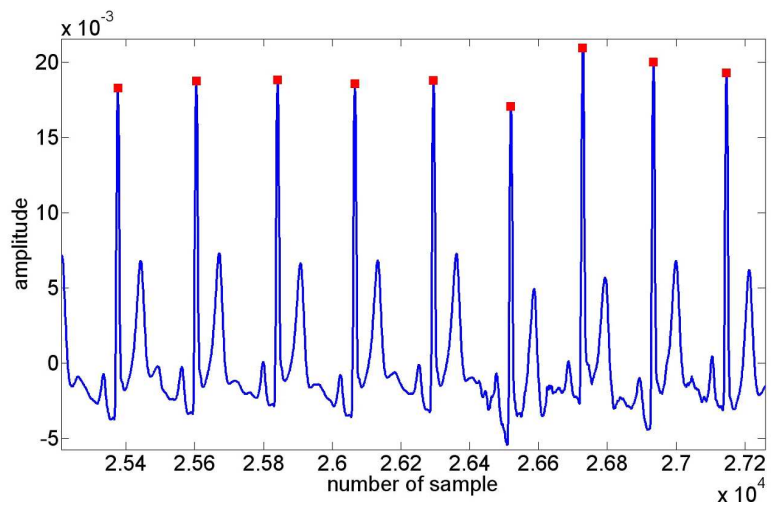

Fig. 4. PQRST complex segmentation - ecg signal with marked $\mathrm{R}$ pointers.

is considered as a formula:

$$
\begin{aligned}
& S N R_{\text {coh }}[d B]=20 \log _{10}\left(S N R_{\text {coh }}\right) \\
& =20 \log _{10}(\sqrt{N})=10 \log _{10}(N),
\end{aligned}
$$

where: $N$ - Number of iterations (number of averaged PQRST complexes)

Coherence condition is performed because of the same length period of averaged complexes. Coherence gain in $\mathrm{dB}$ means power to reduce noisy components and artifacts (sporadically occurred, non-stationary disturbances). The more iterations the higher coherence gain, thus this method can be considered as more efficient than based on correlation selection of the PQRST complex pattern described in others publications.

Example of computed ARP for only 14 iterations $\left(H R=66 \mathrm{bpm}, S N R_{\mathrm{coh}}=11.5 \mathrm{~dB}\right)$ is shown at Fig. 5 .

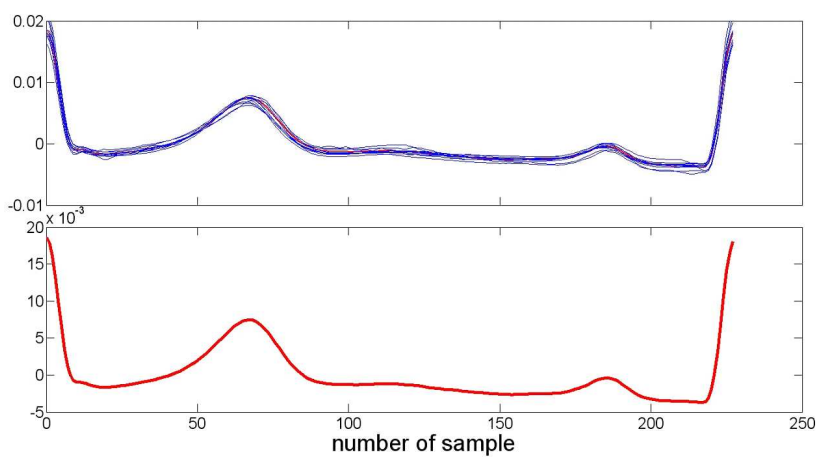

Fig. 5. Accumulated reference pattern (ARF) - cumulative plot (upper plot) and arf after averaging.

After the ARP computation stage, is processed the ARP parameterization stage. Linear Predictive Coefficients are computed using 20-order LPC analysis. Using parametrical prediction analysis we can estimate biological system's transmittance generating PQRST complex based on acquired ECG signal (Fig. 6). 


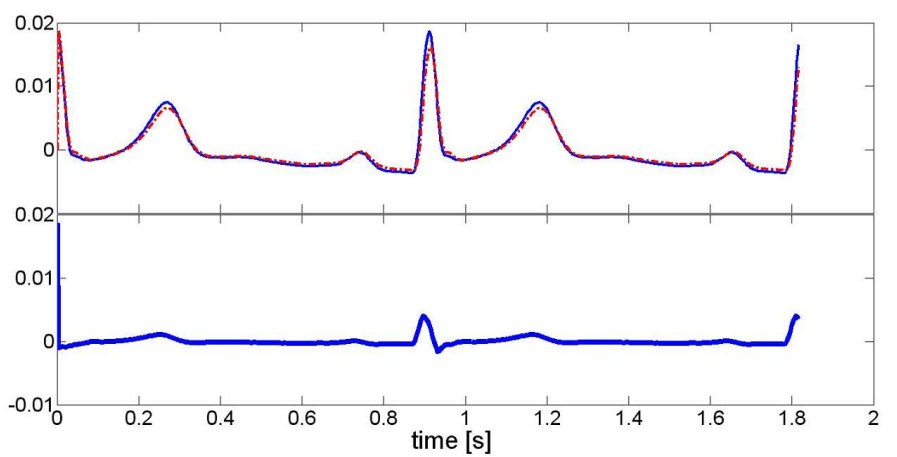

Fig. 6. ARP duplicated 5 times (solid line) and LPC estimate using $20 \mathrm{lpc}$ coefficients (dash-dot line), below prediction error as a residual signal.

\section{Heart Rate Variability module}

Heart Rate Variability (HRV) estimation is based on advanced spectral analysis of electrocardiogram (tachogram of RR values). HRV is noninvasive method of estimation the Sympathetic and Parasympathetic Nervous System influence on the heart rate. HRV spectral analysis is performed based on power Spectral Density estimation using parametric, nonparametric as well as spatial methods. Standard parameters of HRV spectral analysis are computed for dedicated region of interest e.g.: TP - Total Power with DC component, ULF - power in the Ultra Low Frequencies range (0.0005$0.003 \mathrm{~Hz}), \mathrm{VLF}$ - power in the Very Low Frequencies range $(0.003-0.04 \mathrm{~Hz}), \mathrm{LF}$ - power in Low Frequencies range $(0.04-0.15 \mathrm{~Hz}), \mathrm{HF}$ - power in High Frequencies range $(0.15-0.4 \mathrm{~Hz}), \mathrm{LF}_{\text {norm }}$ - normalized power in Low Frequencies range, $\mathrm{HF}_{\text {norm }}$ - normalized power in High Frequencies range, power coefficient $\mathrm{LF} / \mathrm{HF}$, frLF - frequency component possessing maximum power in the LF range, $\mathrm{frHF}$ - frequency component possessing maximum power in the HF range, alpha - spectral tilt for log-log linear interpolating spectrum.

\subsection{HRV_tool - application features}

HRV standard is well described in the scientific literature [13-17]. Application was developed in Matlab environment to verify the HRV algorithms' effectiveness [18]. Graphical User Interface (GUI) is presented at Fig. 7. HRV_t ool uses R-R tachogram as a base for spectral analysis. Cubic Spline approximation is used to receive effect of uniformly sampling tachogram (tachogram is not uniformly sampled function thus is important to ensure uniform sampling) before PSD processing and parameters computation.

Legend:

1. main application's bar: open file, save report, about application,

2. ECG signal's path,

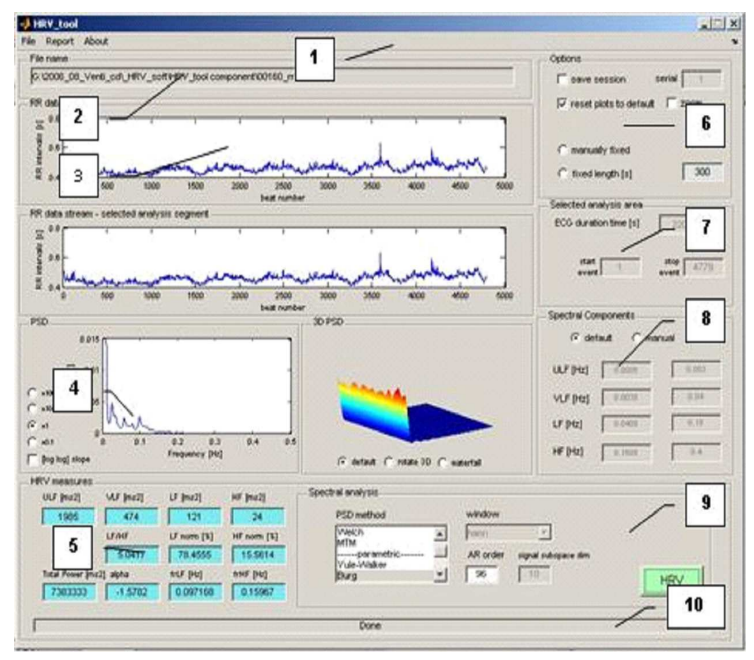

Fig. 7. HRV_tool GUI.

3. display window - RR route (all route and zoom),

4. display window - Power Spectral Density (PSD) and $3 \mathrm{D}$ spectrum,

5. info panel - parameters computed using frequency analysis,

6. options panel — saving and displaying,

7. info panel - R-R tachogram analyzing area,

8. options panel - analyzing frequency bands,

9. options panel - PSD analyzer parameters,

10. info panel - current messages.

Features:

- Short - and long-term HRV spectral analysis

- power spectral density estimation,

- tachogram power computation in the following ranges: ULF, VLF, LF, HF,

- standard coefficients computation: LF/HF, $\mathrm{LF}_{\text {norm }}, \mathrm{HF}_{\text {norm }}, f r L F, f r H F$, alpha,

- power analysis in the chosen regions,

- non-parametrical analysis using methods: periodogram, Welch, MTM,

- parametrical analysis using methods: Yule-Walker, Burg, covariance, modified covariance,

- spatial analysis using Eigenvector method.

- results displaying

- tachogram (R-R periods values),

- scaled tachogram (zoom), 
- PSD,

- spectrogram (time, frequency, amplitude).

- raport generator for chosen by user measurement sessions (*.txt file).

\section{Summary}

ARP can be used as a pattern for human bio signal identification systems, easily stored in medical data bases. It also can be considered as a fingerprint of patient's health state as a one of biological characteristics. Described methods of HR detection and ARP computation are used for military purposes during military aircraft flights to assess the pilot's ability for stress and self control. Robust algorithm against noisy environment, artifacts etc. and relatively easy computation are one of the benefits proposed solution for HR detection problem. HRV tool is a very effective application for HRV frequency analysis when we consider a lot of methods for PSD estimation and precise PSD parameters computation for region of analysis fixed by user.

There are a lot of various algorithms based on ECG signal averaging used for High-Resolution ECG (HRECG) e.g. $[19,20]$. Method described in [19] uses QRS detection algorithm proposed by Pan and Tompkins. The system computes an ECG template by averaging the first 30 consecutive beats approved by user. In $[20]$ is proposed algorithm that estimates the noise variance from the difference signal (difference between averaged beat from the individual beat). In comparison with these methods, proposed SACC method is robust against additive noise. Proposed algorithm is effective for relatively quick ECG beat detection as well as preprocessing stage for searching $\mathrm{R}$ pointers. Together with module mapa01, described HR detection algorithm is also robust against artifacts and non-stationary degradation factors.

\section{References}

[1] B. Surawicz, T. Knilans, Chou's Electrocardiography in Clinical Practice Saunders 2008.

[2] G.S. Wagner, Marriott's Practical Electrocardiography, Publisher Lippincott Williams \& Wilkins, 2007.

[3] J. O'Keefe, S. Hammill, M. Freed, S. Pogwizd, The Complete Guide to ECGs, Jones \& Bartlett Publishers, 2008.

[4] G.D. Clifford, F. Azuaje, P. McSharry, Advanced Methods and Tools for ECG Data Analysis, Artech House Publishers, 2006.
[5] R. Acharya, U, J.S. Suri, J.A.E. Spaan, S.M. Krishnan, Advances in Cardiac Signal Processing, Springer, 2007.

[6] Ch.M. Bishop, Pattern Recognition and Machine Learning, Springer, 2006.

[7] E.N. Bruce, Biomedical Signal Processing and Signal Modeling, Wiley — Interscience, 2000.

[8] F. Kusumoto, Understanding Intracardiac EGMs and ECGs, Wiley-Blackwell, 2009.

[9] K. Goplan, Introduction to Signal and System Analysis, CL-Engineering, 2008.

[10] W. Torbicz, L. Filipczyński, R. Maniewski, M. Nałęcz, E. Stolarski, Biocybernetics and Biomedical Engineering 2000, PAN, Akademicka Oficyna Wydawnicza EXIT, Warsaw 2001, Vol. 2, p. 309.

[11] J. Moczko, L. Kramer, Digital methods of biomedical signal processing, Wydawnictwo Naukowe UAM, Poznań 2001, p. 17.

[12] Z. Piotrowski, K. Różanowski, Effective method of correlation computation Heart Rate in ECG and cumulative reference pattern of the PQRST complex, Research works - Electronics, Vol. 157, Oficyna Wydawnicza Politechniki Warszawskiej, Warsaw 2006, p. 83.

[13] Task Force of The European Society of Cardiology and The North American Society of Pacing and Electrophysiology. Heart rate variability - Standards of measurement, physiological interpretation, and clinical use. European Heart Journal 17, 354 (1996).

[14] P. Augustyniak, Electrodiagnostic signals processing, AGH Uczelniane Wydawnictwa Naukowo-Dydaktyczne, Kraków 2001, p. 174.

[15] T. Krauze, P. Guzik, H. Wysocki, Nowiny Lekarskie. 70, 9, 973, (2001).

[16] T. McAuley, Heart Rate Variability and Ultrafine Particle Exposures, VBM Verlag, 2008.

[17] M. Malik, A.J. Camm, Dynamic Electrocardiography. Wiley-Blackwell, 2004.

[18] Z. Piotrowski, K. Różanowski, Spectral parameters estimation in Heart Rate Variability analysis, Biuletyn Wojskowej Akademii Technicznej, II Konferencja Naukowa Urzadzenia i Systemy Radioelektroniczne, Soczewka $k$. Płocka, 13-15 June 2007, Vol. LVI (2), Warsaw 2007, p. 371.

[19] R.E. Herrera, J.T. Cain, E.G. Cape, G.J. Boyle, A High Resolution ECG Tool for Detection of Atrial and Ventricular Late Potentials, Computers in Cardiology, p. 629.

[20] E. Laciar, R Jane, An Improved Weighted Signal Averaging Method for High-Resolution ECG Signals, Computers in Cardiology, 2001, p. 69, Digital Object Identifier 10.1109/CIC.2001.977593. 